\title{
Ganglion impar radiofrequency ablation for intractable cancer pain: a case report
}

\begin{abstract}
Pelvic and perineal pain are common in patients with malignancy of pelvic organs. Neoplastic invasion of neural tissues and infiltration of organs and sphinters results in severe pain often difficult to manage with pharmacological interventions. Ganglion impar neurolysis is a well documented technique to reduce pain arising from perineal visceral structures such as rectum and anus. We describe the case of a 59 years old woman affected by rectal cancer with drug-refractory severe pain treated with ganglion impar radiofrequency denervation using a trans-sacro-coccygeal approach. The patient reported a significant pain relief lasting up to six months with improved quality of life. Ganglion impar radiofrequency neurolysis is a safe and efficient intervention and should be considered for managing pain arising from pelvic and perineal structures.
\end{abstract}

Keywords: ganglion impar, radiofrequency ablation, neurolysis, pelvic pain, cancer pain, interventional pain management
Volume 2 Issue I - 2018

\author{
Andrea Tinnirello,' Manuel Todeschini,' \\ Francesca Ronconi,' Sandra Barbieri,' Nicola \\ Sbalzer, ${ }^{2}$ Serena Andreoletti' \\ 'Anesthesiology and Pain Management Unit,ASST Franciacorta, \\ Italy \\ ${ }^{2}$ Anesthesiology and Intensive Care Unit,ASST Spedali Civili, \\ Italy
}

Correspondence: Andrea Tinnirello, MD, Anesthesiology and Pain Management Unit, ASST Franciacorta, Italy,Via Don G.Vender 7025127 Brescia, Italy, Tel +393392599568, Email andrea.tinnirello@asst-franciacorta.it

Received: December 15, 2017 | Published: January 12, 2018
Abbreviations: RF, radiofrequency; NRS, numerical rating score; HZ, hertz; A, ampere

\section{Introduction}

Pelvic and perineal pain are common in patients with malignancy of pelvic organs. Neoplastic invasion of neural tissues and infiltration of organs and sphinters results in severe pain whose treatment requires high doses of medications and often results in unsatisfactory pain relief. ${ }^{1}$ Ganglion impar neurolysis is a well documented technique to reduce pain arising from perineal visceral structures such as rectum and anus. ${ }^{1-10}$ Sensitive $\mathrm{C}$ and $\mathrm{A} \delta$ afferent fibers run from visceral structures to the spinal ganglia where the neuron body is located and they run together with the sympathetic fibers innervating splanchnic organs. ${ }^{9}$ Afferent sensitive fibers cross the sympathetic ganglia without synapsing in it, therefore, lesioning the sympathetic chain could result in a reduction of pain transmitted through these somatic fibers. Ganglion Impar is the lowest sympathetic ganglia and is formed by the fusion of the two sympathetic chains that are fused at this level forming a oval-shaped structure.

It is usually located in front of the sacral bone in a midline position under the sacro-coccygeal junction crossed by somatic fibers from the perineum, rectum, anus, distal uretra, vagina, penis and scrotum. ${ }^{11,12}$ Anatomical studies showed variations in the localization of this ganglion between the sacro-coccygeal joint and coccyx end. It is mostly located in front of the sacro-coccygeal joint and coccygeal vertebra. ${ }^{11,12}$ We describe a case report which supports the efficacy and safety of ganglion impar denervation in patients with cancer pain.

\section{Case description}

We describe the case of a 59 years old woman affected by rectal cancer which was diagnosed in April 2016 and involved the rectum from the anal margin for an extension of $7 \mathrm{~cm}$ and extended into the mesorectal lymphonodes withouth metastes (Figure 1). The patient underwent radiation and chemotherapy to reduce cancer mass. After 6 months, though, a full body CT scan showed a reduction of cancer mass into the rectum (which measured $2,5 \mathrm{~cm}$ from the anal margin with no lymphatic involvement) but multiple hepatic metastases were detected. The patient was not proposed for surgery due to the hepatic involvement and was given another chemotherapy cycle comprising cisplatin and fluorouracil. The patient complained of severe pain located in the perianal and perineal region, which was exacerbated by defecation. Basal Numerical Rating Score (NRS) for pain was 8 out of 10 and during defecation the patient reported a NRS of 10 . Pharmacological treatment with dexamethasone, transdermal fentanyl and paracetamol didn't give the patient substantial improvement. The patient was admitted to Palliative Care Department where Pregabalin was added and Fentanyl was replaced with subcutaneous Morphine $100 \mathrm{mg} /$ day. Even with this therapy the patient was still complaining for severe pain located in the perianal region. Rectal exploration was impossible for the extreme pain elicited in the patient by this manouver. After a consultation with the patient's caregiver and the palliative care staff we decided to propose the patient for ganglion impar Radiofrequency (RF) ablation. Written consent was obtained from the patient. The procedure was performed under sterile conditions with fluoroscopic guidance. Antibiotic prophylaxis with intravenous cefazolin $2 \mathrm{~g}$ was given. The patient was prone positioned with a pillow under the iliac crests to reduce lumbar lordosis. Skin was anesthetized with $2 \%$ Lidocaine and a $18 \mathrm{G} 100 \mathrm{~mm}$ long RF needle with an active tip of $5 \mathrm{~mm}$ was inserted under fluoroscopic lateral vision at the sacro-coccygeal junction and the needle tip was positioned under the sacro-coccygeal ligament. Contrast (iopamidol 300 ) was injected and no vascular or epidural absorption was noticed, the contrast spread between the coccyx and the rectum outlining the ganglion impar (Figure 2) Sensory stimulation at $50 \mathrm{~Hz}$ was performed and the patient reported paraesthesia in the perianal region at $0.4 \mathrm{~A}$ intensity.

We excluded involvement of motor fibers with motor stimulation of $1.5 \mathrm{~A}$ at $2 \mathrm{~Hz}$ which didn't elicit any muscle contraction in the perineum or lower limbs. Lidocaine $2 \% 2 \mathrm{cc}$ was then given through the needle to reduce pain related procedure and $\mathrm{RF}$ ablation was performed at $60^{\circ}-65^{\circ}-70^{\circ}-75^{\circ}-80^{\circ} \mathrm{C}$ for 90 seconds each. ${ }^{2}$ Prior to 
needle removal ropivacaine $0.1 \%$ and depomedrol 40 were injected to reduce post procedural pain. The patient was monitored for 4 hours after procedure and no adverse events such as rectal bleeding or neurologic deficits were observed. During the following days the patient reported a substantial imporvement in pain with a NRS of 1 during rest and 3 during defecation. 6 months after procedure patient's conditioned deteriorated and palliative sedation with intravenous morphine was started. Pain was well controlled until her exitus with a basal NRS of 1-2 and 3 during defecation. Morphine was reduced to $40 \mathrm{mg}$ daily after procedure and the dosage was mantained constant except for the last stage.

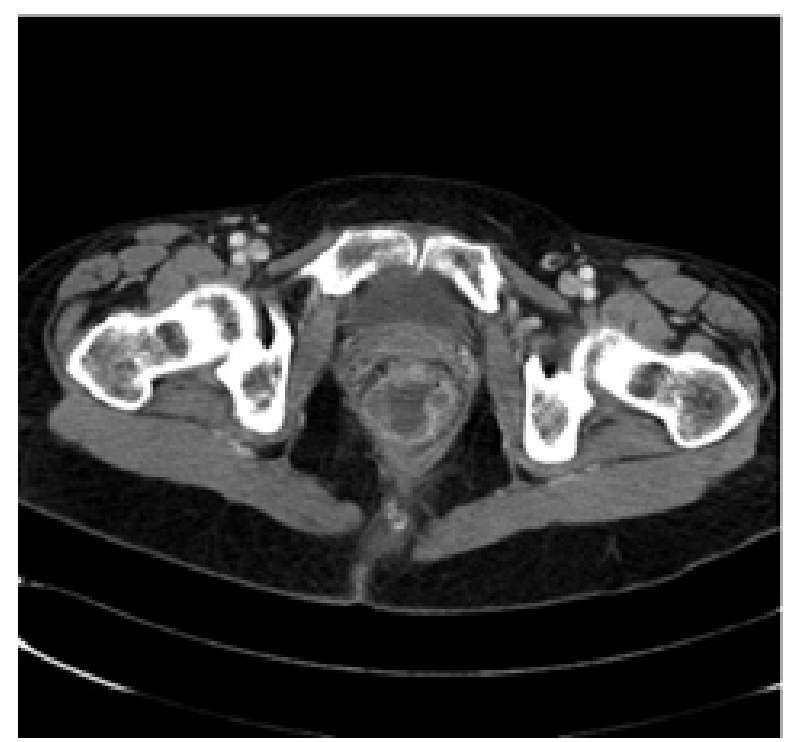

Figure I CT scan showing rectal cancer.

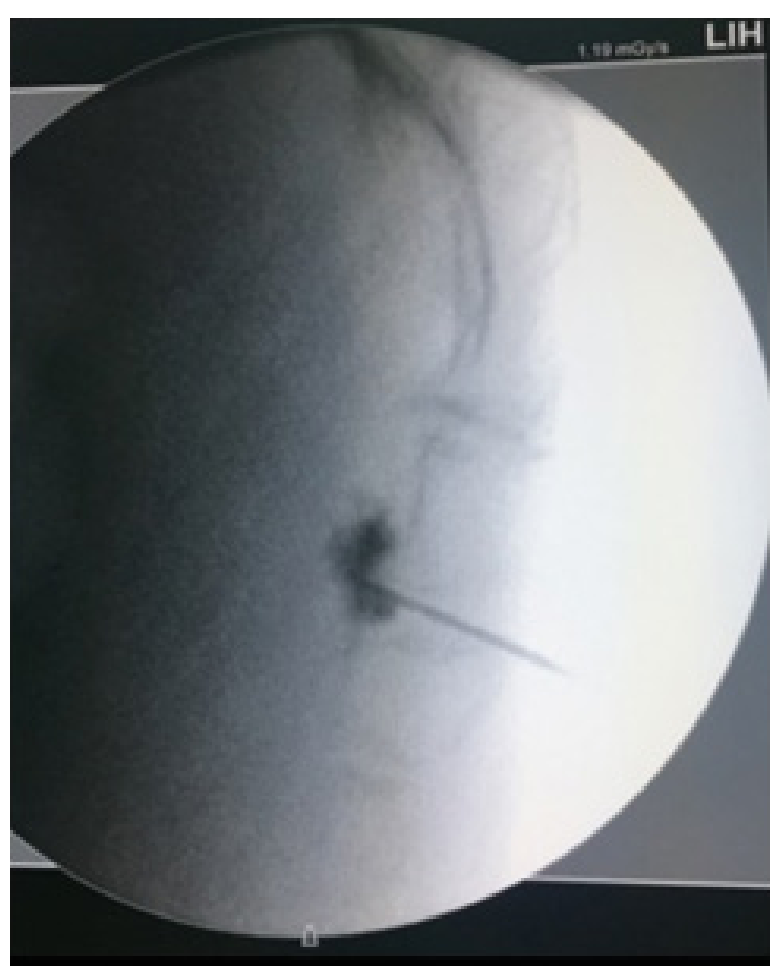

Figure 2 Fluoroscopic lateral vision showing needle positioning at the sacrococcygeal junction and the spread of contrast.

\section{Discussion}

Ganglion impar lesioning was first described by Plancarte in 1990 with a trans-ano-coccygeal approach inserting the needle through the ano-coccygeal ligament. ${ }^{3}$ This approach carries a higher risk of rectal perforation and requires a rectal exploration during needle insertion which can be very painful or even impossible in these patients. We prefer using the trans-sacro-coccygeal approach, as described by Wenn in 1995 which carries lower risk of rectal perforation but can be difficult or impossible in case of a calcified sacro-coccygeal ligament. ${ }^{11}$ Neurolytic techniques are described with the use of agents such as phenol or alcohol.

Due to the relatively high volumes of fluid injected (4 to $6 \mathrm{ml}$ ) these agents carry the risk of lesioning sacral motor fibers. Neurolysis with phenol or alcohol has been reported as efficient in providing pain relief in the short and medium period (up to 6 months). ${ }^{1-6} \mathrm{RF}$ ablation uses a high frequency electrical current which heats the target tissue creating a localized coagulative necrosis. ${ }^{1,2}$ Temperature over $60^{\circ} \mathrm{C}$ is required to create necrosis.

RF procedure contemplate a $50 \mathrm{~Hz}$ sensitive stimulation to ensure that the needle tip is close to the target and a $2 \mathrm{~Hz}$ motor stimulation to exclude proximity of the needle tip to motor fibers. RF has the disadvantage of creating a smaller lesion compared to the spread of neurolytic agents, this is important when the target has a high degree of anatomical variability. However, RF related adverse effects are very unfrequent and less severe compared to neurolytic agents. Ganglion Impar RF ablation has been described by several authors in a relatively small number of patients, for both cancer and non-cancer pain., ${ }^{2,47-10}$ The results are similar to those observed with neurolytic techniques. Dolecek et al. ${ }^{4}$ described 18 patients treated with RF reported a satisfactory pain relief in $57 \%$ of patients at 12 months follow up while Demircay et al. ${ }^{8}$ reported a good pain relief lasting for 6 months in $90 \%$ of 10 patients treated with RF. Adas et al. ${ }^{9}$ described 41 patients treated with RF for coccydynia and reported a successful outcome at 6 months follow up in $90.2 \%$ of patients. ${ }^{9}$ Even if only few studies investigated RF ablation, this technique seems to provide comparable results as chemical neurolysis. The small number of studies and population studied prevents from getting precise data regarding the magnitude and frequency of adverse events with both $\mathrm{RF}$ and neurolysis which appears to be relatively safe.

\section{Conclusion}

Ganglion impar RF ablation is a useful technique for reducing pain arising from pelvic and perineal visceral structures. Anatomical variations of ganlion impar location explains the variations in success of the interventions. ${ }^{14}$ Fluoroscopic guidance is mandatory in order to precisely locate the structure, sensory stimulation is a RF feature that can provide more accurate positioning of the needle tip. A direct comparison between RF ablation and neurolysis would be advisable to determine which treatment could be more durable and efficient in this population of patients.

\section{Acknowledgements}

Andrea Tinnirello: Collected patient data, prepared paper draft.

Sandra Barbieri: Reviewed the literature, Collected patient data, reviewed paper.

Francesca Ronconi: Reviewed the literature, Collected patient data, reviewed paper. 
Serena Andreoletti: Reviewed the literature, Collected patient data, reviewed paper.

Manuel Todeschini: Reviewed the literature, Collected patient data, reviewed paper.

Nicola Sbalzer: Reviewed the literature, Collected patient data, reviewed paper.

\section{Conflict of interest}

Andrea Tinnirello has no conflict of interest to disclosure.

Sandra Barbieri has no conflict of interest to disclosure.

Francesca Ronconi has no conflict of interest to disclosure.

Serena Andreoletti has no conflict of interest to disclosure.

Manuel Todeschini has no conflict of interest to disclosure.

Nicola Sbalzer has no conflict of interest to disclosure.

\section{Patient consent}

Patient's consent for data and images using for research purposes was obtained and recorded in patient's clinical diary.

\section{References}

1. Green IC, Cohen SL, Finkenzeller D, et al. Interventional therapies for controlling pelvic pain: what is the evidence. Curr Pain Headache Rep 2010;14(1):22-32.

2. Gurses E. Impar ganglion radiofrequency application in successful management of oncologic perineal pain. $J$ Pak Med Assoc. 2014;64(6):697-699.

3. Plancarte R, Amescua C, Patt RB, et al. Presacral blockade of the ganglion of walther (ganglion impar). Anesthesiology. 1990;73(3A):748-749
4. Dolecek L, Michalek P, Stern M, et al. Long-term results of radiofrequency thermocoagulation of ganglion impar in perineal pain. Reg Anesth Pain Med. 2008;33(5):197.

5. Scott-Warren JT, Hill V, Rajasekaran A. Ganglion impar blockade: a review. Curr Pain Headache Rep. 2013;7(1):306-311.

6. Malec-Milewska M, Horosz B, Koleda I, et al. Neurolytic block of ganglion of Walther for the management of chronic pelvic pain. Wideochir Inne Tech Malo Inwazyjne. 2014;9(3):458-462.

7. Reig E, Abejon D, del Pozo C, et al. Thermocoagulation of the ganglion impar or ganglion of Walther: description of a modified approach Preliminary results in chronic, nononcological pain. Pain Pract. 2005;5(2):103-110.

8. Demircay E, Kabatas S, Canserver T, et al. Radiofrequency thermocoagulation of ganglion impar in the management of coccydynia: preliminary results. Turk Neurosurg. 2010;20(3):328-333.

9. Adas C, Ozdemir U, Toman H, et al. Transsacrococcygeal approach to ganglion impar: radiofrequency application for the treatment of chronic intractable coccydynia. J Pain Res. 2016;9:1173-1177.

10. Sindt JE, Brogan SE. Interventional treatments of cancer pain. Anesthesiol Clin. 2016;34(2):317-339.

11. Woon JTK, Stringer MD. Clinical anatomy of the coccyx: A systematic review. Clin Anat. 2012;25(2):158-167.

12. Walters A, Muhleman M, Osiro S, et al. One is the loneliest number: a review of the ganglion impar and its relation to pelvic pain syndromes. Clinical Anatomy. 2013;26(7):855-861.

13. Wemm K, Saberski L. Modified approach to block the ganglion impar (ganglion of Walther). Reg Anesth. 1995;20(6):544-545.

14. Oh CS, Chung IH, Li HJ, et al. Clinical implications of topographic anatomy on the ganglion impar. Anesthesiology. 2004;101(1):249-250. 